\title{
Humoral and B-cell memory responses in children five years after pertussis acellular vaccine priming
}

\author{
Maria Carollo a , Elisabetta Pandolfi ${ }^{\mathrm{b}}$, Alberto Eugenio Tozzi ${ }^{\mathrm{b}}$, Anne-Marie Buisman ${ }^{\mathrm{c}}$, \\ Françoise Mascart $^{\mathrm{d}}$, Clara Maria Ausiello ${ }^{\mathrm{a}, *}$ \\ a Anti-Infectious Immunity Unit, Department of Infectious, Parasitic and Immune-mediated Diseases, Istituto Superiore di Sanità (ISS), \\ Viale Regina Elena 299, Rome, Italy \\ ${ }^{\mathrm{b}}$ Epidemiology Unit, Research Center, Ospedale Pediatrico Bambino Gesù, Istituto di Ricovero e Cura a Carattere Scientifico (IRCCS), Piazza S.Onofrio 4, \\ 00165 Rome, Italy \\ ${ }^{\mathrm{c}}$ Centre for Infectious Disease and Control (Cib), National Institute for Public Health and the Environment, Antonie van Leeuwenhoeklaan 9 , \\ 3720 BA Bilthoven, The Netherlands \\ ${ }^{\mathrm{d}}$ Laboratory of Vaccinology and Mucosal Immunity and Immunobiology Clinic- Hôpital Erasme, Université Libre de Bruxelles (U.L.B.), Campus Erasme \\ 808 Route de Lennik, 1070 Brussels, Belgium
}

\section{A R T I C L E I N F O}

\section{Article history:}

Received 11 November 2013

Received in revised form 21 January 2014

Accepted 4 February 2014

Available online 18 February 2014

\section{Keywords:}

Pertussis

Children

Vaccination

Booster vaccination

Antibodies

Memory B-cell immune response

\begin{abstract}
A B S T R A C T
The resurgence of pertussis suggests the need for greater efforts in understanding the long-lasting protective responses induced by vaccination. In this paper we dissect the persistence of humoral and B-cell memory responses induced by primary vaccination with two different acellular pertussis (aP) vaccines, hexavalent Hexavac ${ }^{\circledR}$ vaccine (Hexavac) (Sanofi Pasteur MSD) and Infanrix hexa ${ }^{\circledR}$ (Infanrix) (GlaxoSmithKline Biologicals). We evaluated the specific immune responses in the two groups of children, 5 years after primary vaccination by measuring the persistence of IgG and antibody secreting cells (ASC) specific for vaccine antigens. Part of the enrolled children received only primary vaccination, while others had the pre-school boost dose. A similar level of antigen-specific IgG and ASC was found in Infanrix and Hexavac vaccinated children. The mean IgG levels were significantly higher in children that received the pre-school boost as compared with children that did not receive the boost dose. A longer persistence after the pre-school boost of IgG-Pertussis Toxin (PT) and IgG-pertactin levels was observed in Infanrix primed children, but it was not statistically significant. More than $80 \%$ of children presented a positive ASC B memory response. Around 50\% of children still presented protective IgG-PT levels which are reduced to $36 \%$ in no-boosted children. The pre-school booster dose restores the percentage of protected children above $50 \%$. In conclusion our data underline the importance of giving a booster dose 5 years after primary vaccination and suggest the need for a new vaccine able to induce a long lasting protective response.
\end{abstract}

(C) 2014 Elsevier Ltd. All rights reserved.

\section{Introduction}

The recent resurgence of Bordetella pertussis infections, in spite of the high vaccine coverage in the developed countries, suggests the need of major efforts in the understanding of the long lasting protective responses induced by vaccination. The acquisition

Abbreviations: aP, acellular pertussis; Ab, antibody; ASC, antibody secreting cells; CI, confidence interval; IgG, immunoglobulin G; FBS, foetal bovine serum; FHA, filamentous hemagglutinin; GM, geometric mean; PBMC, peripheral blood mononuclear cells; PBS, phosphate buffered saline; Prn, pertactin; PT, pertussis toxin.

* Corresponding author at: Anti-infectious Immunity Unit, Department of Infectious, Parasitic and Immune-mediated Diseases, Istituto Superiore di Sanità, Viale Regina Elena 299, 00161 Rome, Italy. Tel.: +39 064990 2890; fax: +39 0649903168.

E-mail address: clara.ausiello@iss.it (C.M. Ausiello). of this knowledge will help in defining better vaccination strategies [1-4]. Both infection- and vaccine-induced immunity wanes. Gustafsson and colleagues indicate that protection wanes some 5-7 years after acellular pertussis (aP) vaccination in infancy [5]. The recent California outbreak allowed a more precise quantification of the time of protection induced by aP vaccines. The results showed that protection decreases in a period of five years after the fifth dose, at the age of 5-6 years [3].

In most developed countries aP vaccines are administered in infancy and in pre-school age. Consequently, the peak of incidence of whooping cough has shifted to adolescents and adults $[6,7]$. Although the majority of older age groups suffer from mild symptoms, they are the main source of infection for the not fully vaccinated infants [8] who are at risk for pertussis associated life-threatening complications. During the pertussis-outbreak in 
California, 10 unvaccinated infants died from pertussis disease [3].

The classical approach to evaluate the persistence of immune responses after vaccination or infection is based on the measurement of the antibody levels against the vaccine components, namely pertussis toxin (PT), pertactin (Prn), filamentous hemagglutinin (FHA) and eventually fimbriae. However, the protective immune mechanisms for pertussis are still elusive. Specific serum antibody $(\mathrm{Ab})$ titers are important but not sufficient to represent a correlate of protection $[9,10]$. Since serum Ab levels rapidly wane after vaccination [11-15], cellular immunity such as memory B and $\mathrm{T}$ cells determination can be important parameters to evaluate the protection against whooping cough induced by vaccination [16-21].

In this paper we dissect the B-cell memory responses induced by the primary vaccination with two different aP vaccines, hexavalent Hexavac ${ }^{\circledR}$ vaccine (Hexavac) (Sanofi Pasteur MSD) and Infanrix ${ }^{\circledR}$ hexa vaccine (Infanrix) (GlaxoSmithKline Biologicals), in the 6-7 years of age children groups. We compare the specific immune responses induced by the priming with the two aP vaccines 5 years after primary vaccination, by assessing the persistence of specific $\operatorname{IgG}$, the frequency of B memory cells and the frequency of Ab secreting cells (ASC) specific to the vaccine antigen components. This age group was chosen because it is close to the supposed waning of aP vaccine induced protection [3] and near to the pre-school boost. Since, some of the children surveyed had already received the preschool booster, we could also analyze the impact of primary vaccination on the booster dose of pertussis vaccine.

\section{Materials and methods}

\subsection{Study population, vaccines' information and sample collection procedures}

One hundred and four children were included in the study (Table 1). Sixty-six children received Hexavac and 38 children received Infanrix for all the three doses of the primary vaccination. The vaccine was administered at 3, 5 and 11 months of age [22]. Immune-monitoring was performed when the children were 6-7 year old. All children had a properly completed primary vaccination schedule.

Each $0.5-\mathrm{ml}$ vaccine dose is formulated to contain:

- Hexavac - PT $25 \mu \mathrm{g} ;$ FHA $25 \mu \mathrm{g}$ (http://www.ema.europa. eu/docs/en_GB/document_library/EPAR_-Product_Information/ human/000298/WC500074586.pdf).

- Infanrix - PT $25 \mu \mathrm{g}$; FHA $25 \mu \mathrm{g}$, and Prn $8 \mu \mathrm{g}$ (http://www. ema.europa.eu/docs/en_GB/document_library/EPAR_-_Product Information/human/000296/WC500032505.pdf).

As indicated in Table 1 part of the children already received the pre-school booster before the immune monitoring; the vaccine used was Boostrix ${ }^{\circledR}$ (GlaxoSmithKline): PT toxoid $8 \mu \mathrm{g}$; FHA $8 \mu \mathrm{g}$ and $\operatorname{Prn} 2.5 \mu \mathrm{g}$.

This study was conducted in accordance with the Declaration of Helsinki (1964). Ethics approval was obtained by the Bambino Gesù paediatric hospital, and the children's parents provided a written informed consent.

Seven milliliters of peripheral blood in sodium heparin were collected for B-cell memory response and serological analysis.

Discrepancies in the number of children enrolled and the number of sample tested in the ASC assay, as indicated in figures, are due to insufficient number of peripheral blood lymphocytes; the pertussis antigen priority was PT $>$ FHA $>$ Prn.

\subsection{Reagents, antigens and mitogen}

Genetically detoxified PT and FHA antigens were purified starting from crude material obtained from Novartis, Siena, Italy, as described in [23]. Prn was expressed and purified from an Escherichia coli construct as described in [24]. Tetanus toxoid ( $4200 \mathrm{Lf} / \mathrm{ml}$ Novartis) was used as positive control of B memory responses.

\subsection{Pertussis specific serology}

For measurement of plasma IgG levels specific for PT, Prn and FHA antigens the fluorescent bead-based multiplex immunoassay was used as described in Ref. [19,23-25]. In the absence of wellestablished serological markers for protection against pertussis, arbitrary level of $\geq 20 \mathrm{EU} / \mathrm{ml}$ for the IgG-PT was defined as protective [26-28].

An IgG-PT value above $100 \mathrm{EU} / \mathrm{ml}$ was considered indicative of a recent $B$. pertussis infection, unless the children had the booster less than a year before $[28,29]$. We found one no-boosted child in the Hexavac group and one boosted child in the Infanrix group with IgG-PT levels above $100 \mathrm{EU} / \mathrm{ml}$. Considering that we focused on the persistence of vaccine induced immunity, these children were removed and not considered for the further analysis in all assays performed.

\subsection{Pertussis specific B-cell memory responses}

Peripheral blood mononuclear cells (PBMC) were isolated and frozen as previously described [19]. On the day of the experiment, the vial was quickly thawed at $37^{\circ} \mathrm{C}$, cells washed in RPMI-1640, counted and suspended in AIM-V ${ }^{\circledR}$ (Gibco) at the final concentration of $2 \times 10^{6}$ cells $/ \mathrm{ml}$. Medium was supplemented with $10 \%$ FBS (Hyclone), human CpG ( $3 \mu \mathrm{g} / \mathrm{ml}$ from Eurogentec, Seraing, Belgium), human IL-10 and IL-2 (both $10 \mathrm{ng} / \mathrm{ml}$, Peprotech, Milan, Italy), PT, FHA, and Prn at $10 \mathrm{ng} / \mathrm{ml}$ each. Cells were cultured 5 days at $37^{\circ} \mathrm{C}$ in a $5 \% \mathrm{CO}_{2}$ incubator.

96-wells high-protein-binding MultiScreen Immobilon-P Membrane plates (MAIPS10, Millipore, UK) were pre-wetted with $35 \%$ ethanol $(15 \mu \mathrm{l} /$ well/60 s), washed three times with sterile PBS, coated with $50 \mu \mathrm{l} /$ well of $20 \mu \mathrm{g} / \mathrm{ml}$ of FHA, $30 \mu \mathrm{g} / \mathrm{ml}$ of PT or Prn, $7 \mathrm{Lf}$ of tetanus toxoid in sterile PBS or with goat anti-human IgG $(2 \mu \mathrm{g} / \mathrm{ml}$, Dako) as a positive control, and left overnight at $4{ }^{\circ} \mathrm{C}$. Plates were blocked (AIM-V ${ }^{\circledR}+10 \%$ FBS, $2 \mathrm{~h}$, $37^{\circ} \mathrm{C}$ ) and $2 \times 10^{5}$ cells/well cultured cell suspensions were incubated in duplicate wells overnight. Plates were washed with $0.05 \%$ Tween-20 in PBS (PBS-T) and incubated with alkaline phosphatase-conjugated goat anti-human IgG (Southern Biotechnologies, UK) for $2 \mathrm{~h}$ at $37^{\circ} \mathrm{C}$. After incubation, plates were washed with PBS-T followed by washing with PBS and developed with $50 \mu \mathrm{l} /$ well of a ready-to-use substrate solution of 5-bromo-4chloro-3-indolylphosphate/nitrobluetetrazolium (BCIP/NBT, KPL, USA) for 5-10 min at room temperature. Plates were washed with tap water and dry. Spots, representing single ASC, were counted using the ELISPOT Reader (Eli.Expert, the A.EL.VIS Thema, Bologna, Italy) and expressed as ASC specific to the indicated antigen normalized to total IgG ASC. The phenotype of the expanded B cells was analyzed, both at day 0 and after 5 days of culture, by flow cytometry using CD19-PE, CD27-FITC, CD3PcP monoclonal antibodies (all purchased from BD Biosciences, San Jose, CA). A response was defined positive when spots $(A S C \geq 1)$ were present in the wells coated with antigen as compared to not-coated control wells that were negatives. 
Table 1

Study population: base-line characteristic of the cohort.

\begin{tabular}{|c|c|c|c|c|c|c|c|}
\hline \multirow[t]{2}{*}{ Primary vaccination } & \multirow[t]{2}{*}{$\begin{array}{l}\text { Number of children } \\
\text { enrolled }\end{array}$} & \multirow[t]{2}{*}{$\operatorname{Sex}(F / M)$} & \multirow[t]{2}{*}{ Age (years) } & \multirow[t]{2}{*}{$\begin{array}{l}\text { Number of children } \\
\text { no boosted }\end{array}$} & \multirow[t]{2}{*}{$\begin{array}{l}\text { Number of children } \\
\text { boosted [Boostrix] }\end{array}$} & \multicolumn{2}{|c|}{$\begin{array}{l}\text { Suspected pertussis } \\
>100 \mathrm{EU} / \mathrm{ml}>12 \text { months } \\
\text { from the boost }\end{array}$} \\
\hline & & & & & & No boost & Boost \\
\hline Hexavac & 66 & $26 / 40$ & $6-7$ & 24 & 41 & 1 & \\
\hline Infanrix & 38 & $18 / 20$ & $6-7$ & 10 & 27 & & 1 \\
\hline
\end{tabular}

\subsection{Statistical analysis}

Statistical analysis was performed using the GraphPad Prism ${ }^{\circledR}$ software (San Diego, CA, USA). The results are expressed as geometric mean (GM) with 95\% confidence interval (CI). To compare differences between groups two-sided Mann-Whitney test was performed. To compare differences within groups Wilcoxon pairedsamples test was performed. To analyze the correlation between variables Spearman rank correlations and linear regression analysis were used. $p<0.05$ was considered statistically significant.

\section{Results}

\subsection{Pertussis specific serology}

Sixty-six children primed with Hexavac and 38 children primed with Infanrix were included in the study (Table 1). IgG-PT and IgGFHA levels were comparable in the two cohorts. IgG-Prn levels were significantly higher in Infanrix than in Hexavac primed groups (Fig. 1A).

We found a significantly higher level of IgG to all pertussis antigens in children tested after the booster dose as compared to no-boosted in both children groups (Fig. 1B). Moreover, a higher level of IgG-Prn was found in the Infanrix- than in Hexavac-primed group, in the boosted children (Fig. 1B). This result is probably due to the absence of Prn in the Hexavac vaccine and consequently the absence of a recall response due to Prn after the boostrix vaccination in Hexavac primed children.

When the data were analyzed in term of the presence of protective Ab levels, we found that around $50 \%$ of children presented levels of IgG-PT higher than $20 \mathrm{EU} / \mathrm{ml}$, an arbitrary protective $\mathrm{Ab}$ level [26-28] (Table 2). The percentage of positive responses was lower in children who did not receive a booster dose as only $36.4 \%$ of them still had protective IgG-PT levels (44.4\% Infanrix vs $33.3 \%$ Hexavac). The proportion of positive responses increased after the booster dose to $63.2 \%$ ( $74.1 \%$ Infanrix vs $56.1 \%$ Hexavac). No significant differences between the two groups were found, even if a higher proportion of Infanrix-vaccinated children had protective IgG-PT levels compared to Hexavac-primed children (Table 2).

In the cohort of boosted children, the time between the booster vaccination and the blood sampling to perform the $B$ response assays was variable from few days to more than one year. The IgGPT and IgG-Prn concentrations were plotted by six months intervals considering for each child the time elapsed from the booster and the blood sampling (Fig. 2). In Hexavac-primed children group, a significant increase of IgG-PT levels, was found in the 0-6 months $(p=0.014)$ and in the $7-12$ months $(p=0.011)$ intervals in the boosted as compared to no-boosted children. A significant decrease

Table 2

Percentage of children with anti PT titers considered protective IgG-PT $>20 \mathrm{EU} / \mathrm{ml}$.

\begin{tabular}{llll}
\hline Primary vaccination & All children & No boosted & Boosted [Boostrix] \\
\hline All children & 54.5 & 36.4 & 63.2 \\
Hexavac & 47.7 & 33.3 & 56.1 \\
Infanrix & 66.7 & 44.4 & 74.1 \\
\hline
\end{tabular}

of the IgG-PT level was found when comparing the IgG-PT at 13-20 vs $0-6(p=0.024)$ and vs $7-12(p=0.009)$ months intervals (Fig. $2 \mathrm{~A})$. In Infanrix-primed children, the IgG-PT antibody responses significantly increased in the $0-6$ months $(p=0.049)$ interval in the boosted as compared to no-boosted children group and tended to persist longer, although not significantly (Fig. 2A).

When the two vaccine-primed groups were compared, a significantly higher IgG-PT level was found at 13-20 months interval $(p=0.006)$ in Infanrix- compared to Hexavac-primed group (Fig. 2A).

The effect of the booster vaccination on the IgG-Prn levels was clear only in Infanrix group (0-6 months group vs no-boosted group $p=0.020$ ) (Fig. 2B). This is in line with the fact that Prn is not present in the Hexavac vaccine. The levels of IgG-Prn were higher in Infanrix primed children as compared to Hexavac, statistical significance being reached in the no-boosted $(p=0.025)$ and in 0-6 months interval $(p=0.036)$ in boosted groups. In Hexavacprimed group, a significant increase of IgG-Prn levels was found in 13-20 months group but it did not appear to be linked to the pertussis booster vaccination, being Prn not present in the vaccine. The decrease of IgG-Prn levels respect the time elapsed was not observed.

The IgG-FHA levels analysis indicated a trend for higher IgG levels in Hexavac- as compared to Infanrix-primed children in the group of 0-6 months, even if the differences were not significant (not shown). Moreover the effect of the booster vaccination was limited and the decrease of IgG-FHA levels respect the time elapsed was not observed (not shown).

\subsection{Pertussis specific B-cell memory}

We analyzed the frequency of memory B cells, defined as CD3-, CD19+, CD27+ in the two groups of children primed with the two different aP vaccines. The memory B-cell population increased in average 4-fold during the five days of culture. We did not find significant differences in the two groups of children respect to the type of vaccine used in the primary vaccination (not shown).

In order to explore the specificities of the B-cell memory pool, the frequency of ASC for pertussis antigens was evaluated.

The frequency of total IgG ASC and tetanus ASC, internal control, were similar, irrespective of the vaccine used, indicating that the two groups were comparable (not shown).

The number of ASC specific for PT, Prn and FHA, normalized to total IgG ASC, is shown in Fig. 3A. No significant differences were found in the two groups of children. No other differences were found also when the children were grouped considering whether they received the pre-school booster or not (Fig. 3B), even when analyzed by 6 months interval (data not shown). Almost all children presented a positive response (ASC $\geq 1$ ) to PT (88.6\% Infanrix vs $83.7 \%$ Hexavac). We were not able to see an increase of specific ASC because the post boost assay was not planned in an optimal time-interval after the vaccine boost dose [17].

When the correlation analysis between IgG levels and ASC was performed no correlation was found when PT or FHA antigens were considered, while a low correlation was found for Prn antigen both in all children and in the boosted group in 
A

PT

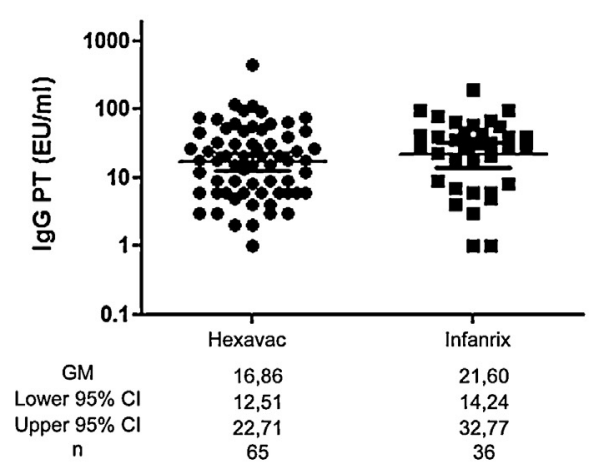

PT

B

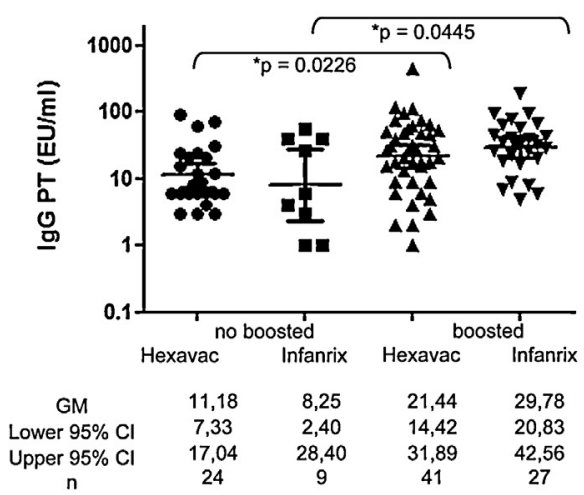

Prn

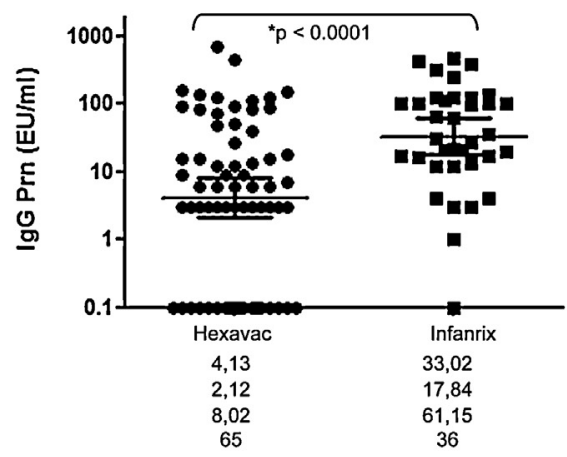

Prn

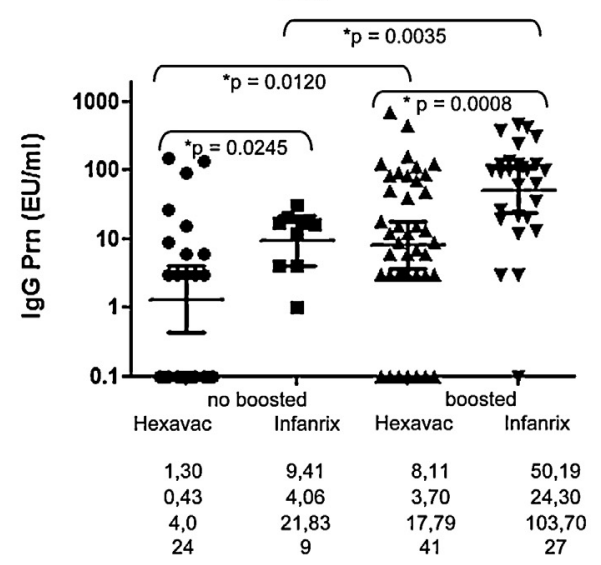

FHA
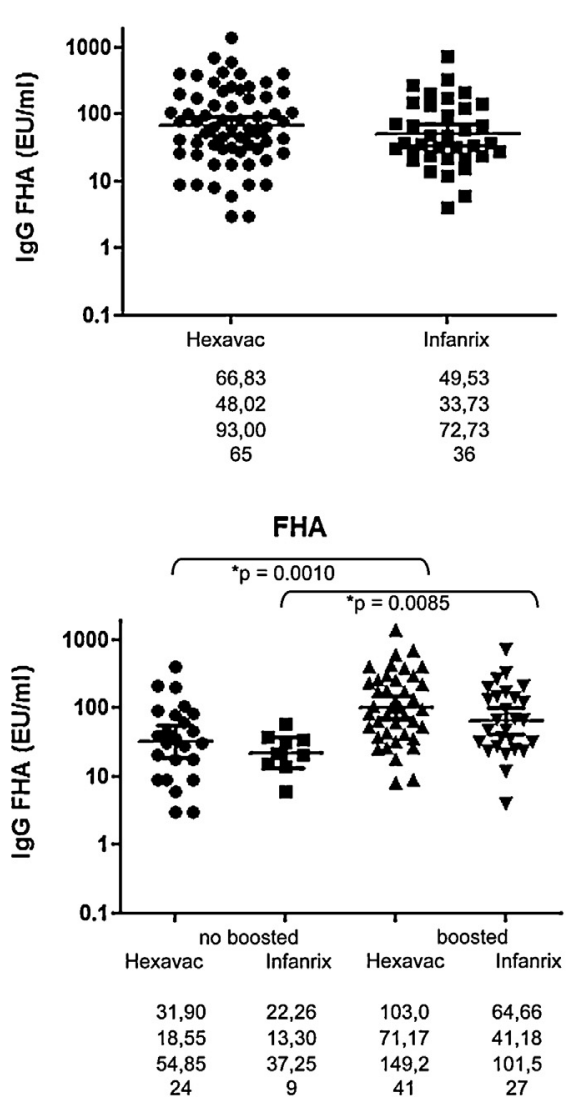

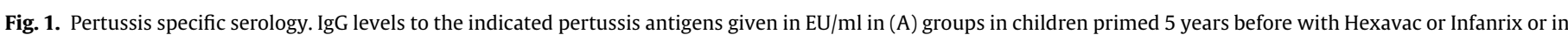

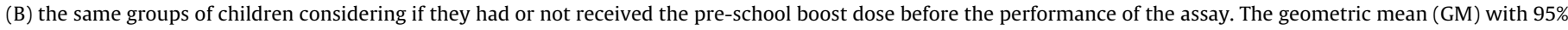

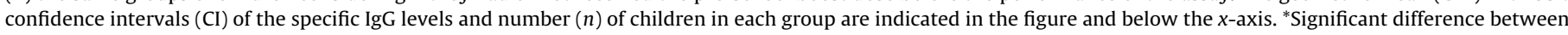
the groups is indicated.

Hexavac (all children $r=0.4556, p=0.0017$; boosted children $r=0.5075, p=0.005$ ) and Infanrix (all children $r=0.45, p=0.0086$; boosted children $r=0.5489, p=0.0045$ ) primed children.

\section{Discussion}

Pertussis resurgence, especially among adolescents and adults, is due to the decrease of the protection induced by vaccination with aP vaccines $[1,30,31]$. In our settings we found, that primary vaccination, performed five years before with the two aP vaccines, results in similar responses respect to Ab levels, expansion of memory B cells and ASC specific for pertussis vaccine antigens in the two cohorts of children. More than $80 \%$ of children presented a positive ASC B memory response, indicating the existence of pertussis specific responses induced by the primary vaccination in the great majority of children. We found that around $50 \%$ of children still presented IgG-PT levels higher than $20 \mathrm{EU} / \mathrm{ml}$, which is assumed to be protective. This percentage was reduced to $36.4 \%$ in the no-boosted children, and reached $63.2 \%$ in the cohort of boosted children, thus showing the importance of giving a booster dose 5 years after primary vaccination.

However it is not yet known if the levels higher than $20 \mathrm{EU} / \mathrm{ml}$ are truly protective. In recent epidemiological study, Witt and colleagues found that the current schedule of aP vaccine doses is insufficient to prevent outbreaks of pertussis and the authors noted a markedly increased rate of disease from the ages 8 to 12 years [31]. Thus presumably the levels of IgG-PT at six years can be still considered protective. From the Italian epidemiological data no indication of a major incidence of pertussis in this class of age was found [32].

We observed that the levels of Ab specific for the PT and Prn in children primed with Infanrix persist longer after the boost than in children primed with Hexavac. In the case of IgG-Prn levels, a significant difference in the post-boost between Infanrix- and Hexavac-priming was observed (Fig. 1). These results are not surprising, considering that in the Hexavac vaccine the Prn antigen is not present and this may explain the lower power of recall made by the Boostrix vaccine in Hexavac primed children.

The decrease over the time of the $\mathrm{Ab}$ responses was quite limited in the case of Prn and FHA antigens. Moreover a clear response to Prn was also observed in not boosted Hexavac primed children. All this can be an indication of a natural booster due to cross-reactive bacteria or to the encounter with other Bordetellea species.

Less clear is the higher persistence of IgG-PT response in children primed with Infanrix compared with Hexavac (Fig. 2A). These data may indicate that the booster response is more efficient in children originally vaccinated with Infanrix and this observation reveals potential differences in long-term protection between the two vaccines, perhaps due to differences in their vaccine composition or preparation procedures.

In both vaccines PT is present and is chemically inactivated. In a recent study [33] the immune responses against $B$. pertussis after immunization of mice with Infanrix and Pediacel (Sanofi-Pasteur) showed similar protection but the levels of $\mathrm{Ab}$ to vaccinal antigens were different. The authors concentrated their explanation on the differences in the two different adjuvants, with lower Ab levels 
Hexavac

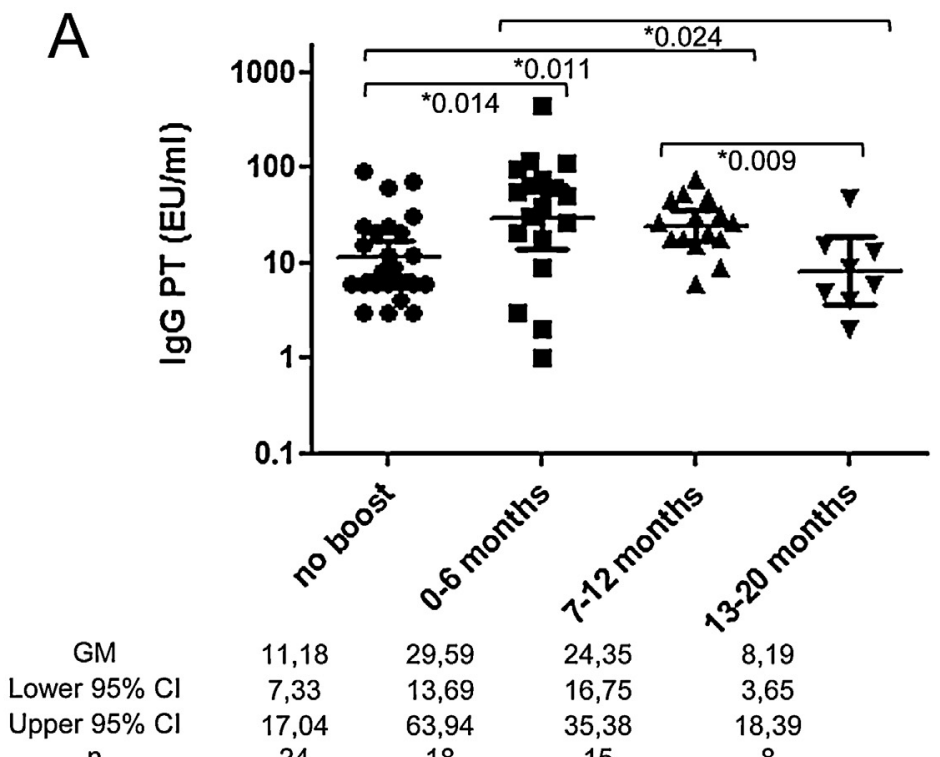

Infanrix

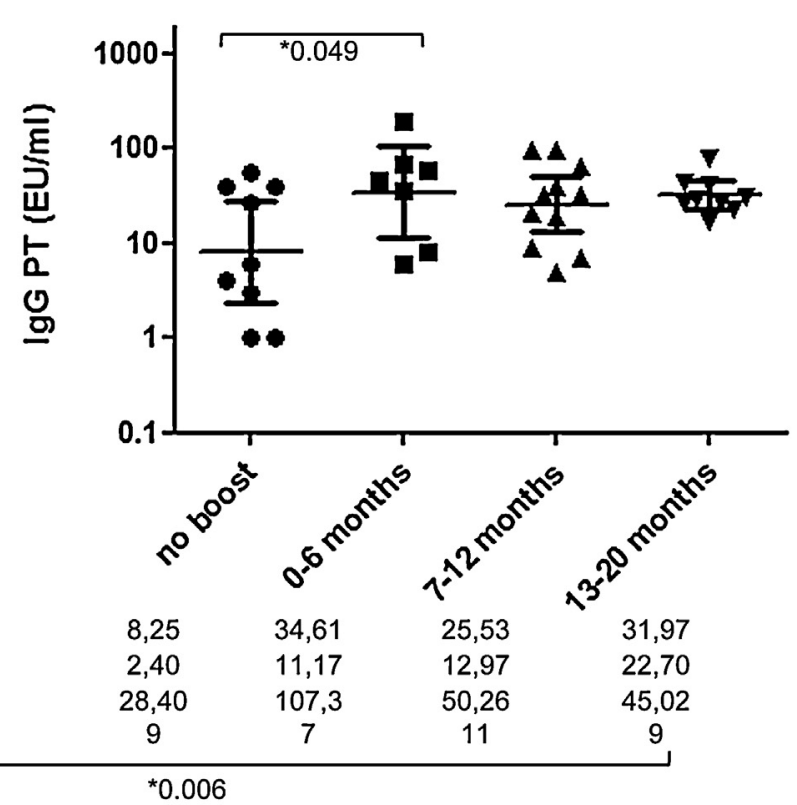

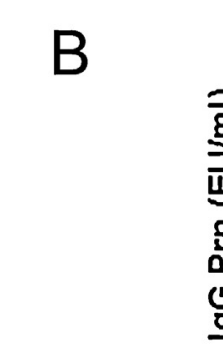

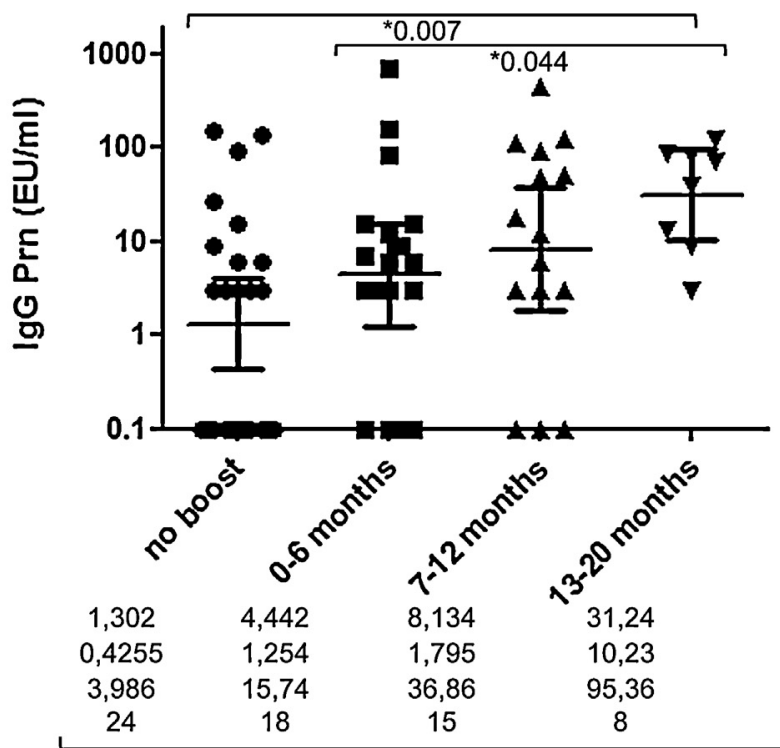

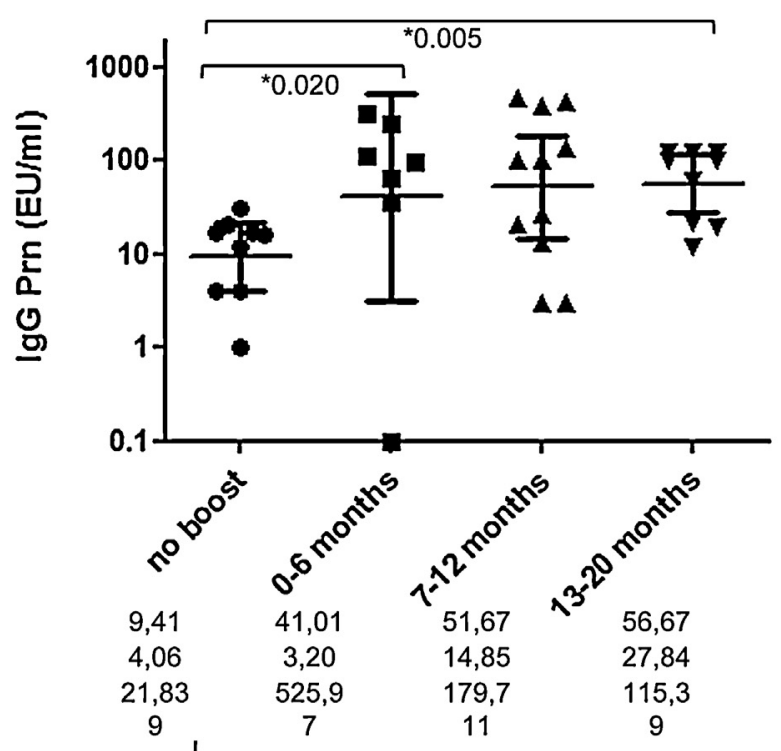

${ }^{*} 0.036$

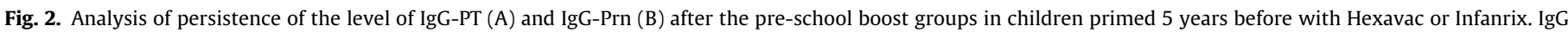

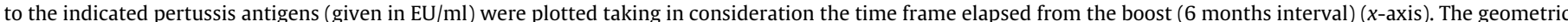

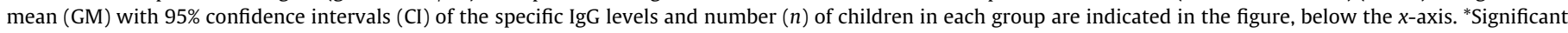
difference between the groups is indicated.

induced by aluminum phosphate-adsorbed compared to aluminum hydroxide-adsorbed PT, FHA and Prn [34]. In our case, this explanation is not appropriate because both vaccines are formulated using antigens adsorbed onto aluminum hydroxide, even if in Infanrix the content of aluminum hydroxide is higher than in the Hexavac ( 0.5 vs $0.3 \mathrm{mg}$ ).

We cannot exclude a role of other excipients in the vaccine preparations or in the chemically inactivation procedures. In Infanrix, PT is inactivated with treatment with glutaraldehyde and formaldehyde while in Hexavac only with glutaraldehyde. It is not clear if these differences may affect the antigenic power of the vaccines, however, in recent studies, Infanrix vaccine priming demonstrated a greater ability to induce an antibody response for the hepatitis component, greater than the Hexavac vaccine [22] even if the memory $B$ and $T$ cell responses were fully comparable $[35,36]$.

When the levels of responses after the pre-school booster vaccination were considered, our data are only partially in agreement with those from previous authors [16-18], however we need to take into account that, following the Italian National vaccine calendar (http://www.salute.gov.it/imgs/C_17_pubblicazioni_1721_allegato. pdf), the pre-school booster is the fourth dose of vaccine in Italy 
A

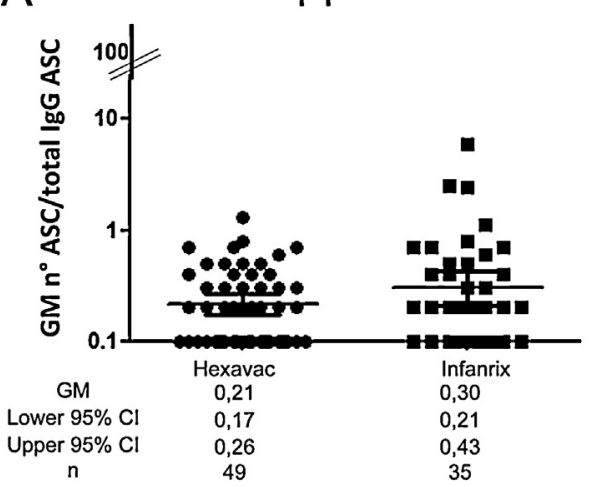

B

\section{B}

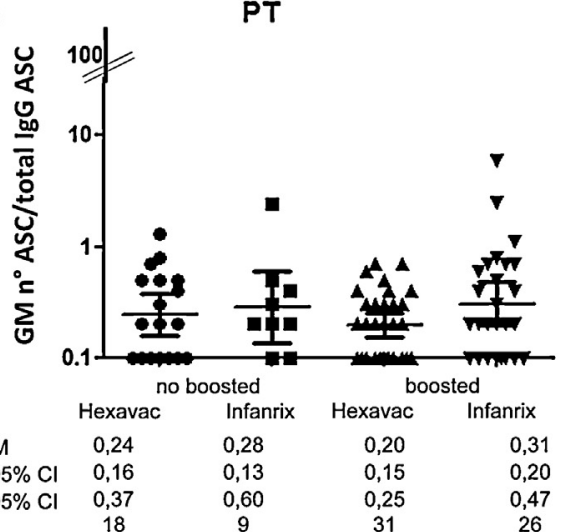

Prn

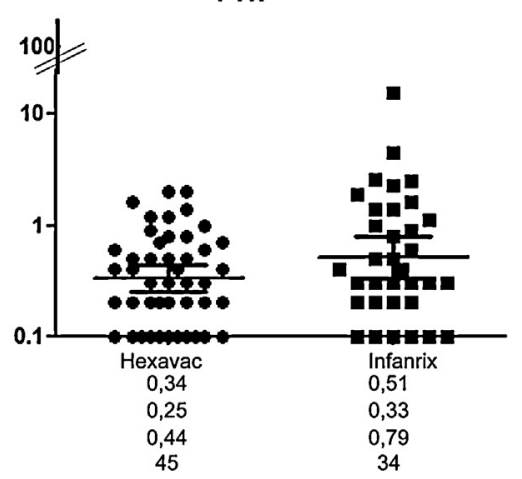

Prn

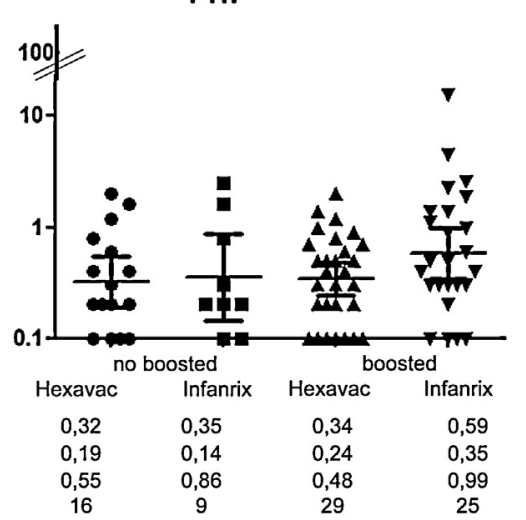

FHA

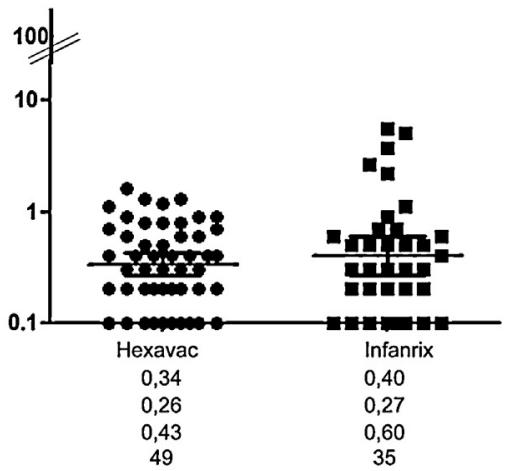

FHA

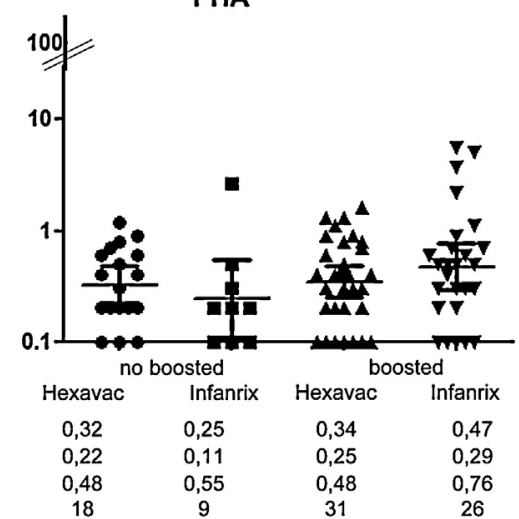

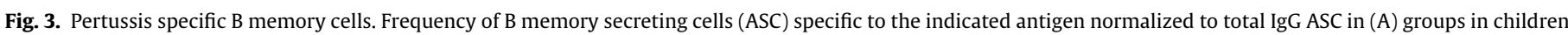

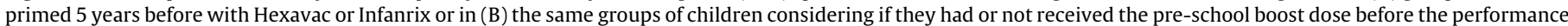
of the assay. Geometric mean (GM) of ASC with $95 \%$ confidence intervals (CI) and number $(n)$ of children in each group are indicated below the $x$-axis.

and not the fifth dose as in several other developed countries [37]. Schure and colleagues [18] evaluated the boost response at 28 days and 2 years after boost in a population of 4 -year-old children. On average, the Ab response measured in our study is lower compared to that measured in the Shure's study at 28 days but is higher to levels recorded after 2 years. Furthermore, in our study the time elapsed since the boost to the Ab test is greatly variable, ranging between a few days up to almost 2 years, while in Schure's study Ab determination is scheduled at well-fixed time.

In recent papers $[16,17]$ the authors found that the number of PT- and Prn-specific ASC at one month post-booster significantly correlate with the corresponding IgG level. We found a correlation only for Prn-specific ASC and the corresponding IgG level. We did not find a correlation with the PT response. We have no specific explanation, but again the time after the booster and the measurement of the B response is variable and, in some cases, the time between the booster and the test may be too long, when the Ab levels are already vanished. Furthermore, the number of children tested after the booster in the 0-6 months interval group is probably not sufficient to have a significant correlation between the IgG-PT and the ASC PT data.

In conclusion our data underline that (i) the percentage of subjects with anti PT antibody titers above the level considered to be protective, after five years from the primary vaccination, is below $50 \%$, (ii) the pre-school booster restores the percentage of protected children above $50 \%$ (Table 2), indicating the importance of the booster vaccination, and (iii) post booster persistence may be different depending on the type of vaccine used for the primary vaccination.
Due to limitation of our study we cannot give conclusions on the persistence of protective responses. Boosting by natural infection could be a major consideration, and it will be very difficult to differentiate between what is due to vaccination, and what is due to infection unless carefully monitored. Our data support the need of sponsoring an ad hoc planned study to answer these specific limitations. Moreover these data support the need to give greater insight in memory immunity studies, important for future pertussis vaccine design as well as for future decisions concerning pertussis vaccination policies to sustain long-lasting protective levels of immunity, still not reached by the vaccine on the market nowadays.

\section{Acknowledgements}

The authors thank all of the children and their families, whose collaboration made this study possible. We thank Raffaella Palazzo, Manuela Bianco and Kemal Ozturk for technical support. We thank Giorgio Fedele for critically reading the manuscript. This study was supported by grants from the European Commission under grant agreement \#201502 (ChildINNOVAC) and by the Italian Ministry of Health (CCM 1M51 and RF-2010-317709-V59). The funders had no role in study design, data collection and analysis, decision to publish, or in preparation of the manuscript.

\section{References}

[1] Zepp F, Heininger U, Mertsola J, Bernatowska E, Guiso N, Roord J, et al. Rationale for pertussis booster vaccination throughout life in Europe. Lancet Infect Dis 2011;11:557-70. 
[2] Cherry JD. Epidemic pertussis in 2012. The resurgence of a vaccine-preventable disease. N Engl J Med 2012;367:785-7.

[3] Klein NP, Bartlett J, Rowhani-Rahbar A, Fireman B, Baxter R. Waning protection after fifth dose of acellular pertussis vaccine in children. N Engl J Med 2012;367:1012-9.

[4] Jefferson T, Rudin M, DiPietrantonj C. Systematic review of the effects of pertussis vaccines in children. Vaccine 2003;21:2003-14.

[5] Gustafsson L, Hessel L, Storsaeter J, Olin P. Long-term follow-up of Swedish children vaccinated with acellular pertussis vaccines at 3, 5, and 12 months of age indicates the need for a booster dose at 5 to 7 years of age. Pediatrics 2006;118:978-84.

[6] Hallander HO, Andersson M, Gustafsson L, Ljungman M, Netterlid E. Seroprevalence of pertussis antitoxin (anti-PT) in Sweden before and 10 years after the introduction of a universal childhood pertussis vaccination program. APMIS 2009; 117:912-22.

[7] Quinn HE, McIntyre PB, Backhouse JL, Gidding HF, Brotherton J, Gilbert GL. The utility of seroepidemiology for tracking trends in pertussis infection. Epidemiol Infect 2010;138:426-33.

[8] de Greeff SC, Mooi FR, Westerhof A, Verbakel JM, Peeters MF, Heuvelman CJ, et al. Pertussis disease burden in the household: how to protect young infants. Clin Infect Dis 2010;50:1339-45.

[9] Storsaeter J, Hallander HO, Gustafsson L, Olin P. Levels of anti-pertussis antibodies related to protection after household exposure to Bordetella pertussis. Vaccine 1998;16:1907-16.

[10] Cherry JD, Gornbein J, Heininger U, Stehr K. A search for serologic correlates of immunity to Bordetella pertussis cough illnesses. Vaccine 1998;16:1901-6.

[11] Edelman K, He Q, Mäkinen J, Sahlberg A, Haanperä M, Schuerman L, et al. Immunity to pertussis 5 years after booster immunization during adolescence. Clin Infect Dis 2007;44:1271-7.

[12] Riffelmann M, Littmann M, Hülsse C, Wirsing von König CH. Antibody decay after immunisation of health-care workers with an acellular pertussis vaccine. Eur J Clin Microbiol Infect Dis 2009;28:275-9.

[13] Hallander HO, Gustafsson L, Ljungman M, Storsaeter J. Pertussis antitoxin decay after vaccination with DTPa. Response to a first booster dose $31 / 2-61 / 2$ years after the third vaccine dose. Vaccine 2005;23:5359-64.

[14] Giuliano M, Mastrantonio P, Giammanco A, Piscitelli A, Salmaso S, Wassilak SG. Antibody responses and persistence in the two years after immunization with two acellular vaccines and one whole-cell vaccine against pertussis. J Pediatr 1998;132:983-8.

[15] Cassone A, Ausiello CM, Urbani F, Lande R, Giuliano M, La Sala A, et al. Cell-mediated and antibody responses to Bordetella pertussis antigens in children vaccinated with acellular or whole-cell pertussis vaccines. The Progetto Pertosse-CMI Working Group. Arch Pediatr Adolesc Med 1997;151:283-9.

[16] Hendrikx LH, de Rond LG, Oztürk K, Veenhoven RH, Sanders EA, Berbers GA, et al. Impact of infant and preschool pertussis vaccinations on memory B-cell responses in children at 4 years of age. Vaccine 2011;29:5725-30.

[17] Hendrikx LH, Felderhof MK, Oztürk K, de Rond LG, van Houten MA, Sanders EA, et al. Enhanced memory B-cell immune responses after a second acellular pertussis booster vaccination in children 9 years of age. Vaccine 2011;30: 51-8.

[18] Schure RM, Hendrikx LH, de Rond LG, Oztürk K, Sanders EA, Berbers GA, et al. Differential T- and B-cell responses to pertussis in acellular vaccine-primed versus whole-cell vaccine-primed children 2 years after preschool acellular booster vaccination. Clin Vaccine Immunol 2013;20:1388-95.

[19] Carollo M, Palazzo R, Bianco M, Smits K, Mascart F, Ausiello CM. Antigen-specific responses assessment for the evaluation of Bordetella pertussis T cell immunity in humans. Vaccine 2012;21:1667-74.
[20] Smits K, Pottier G, Smet J, Dirix V, Vermeulen F, De Schutter I, et al. Different $\mathrm{T}$ cell memory in preadolescents after whole-cell or acellular vaccination. Vaccine 2013;32:111-8.

[21] Bernasconi NL, Traggiai E, Lanzavecchia A. Maintenance of serological memory by polyclonal activation of human memory B cells. Science 2002;298:2199-202.

[22] Zanetti AR, Romanò L, Giambi C, Pavan A, Carnelli V, Baitelli G, et al. Hepatitis B immune memory in children primed with hexavalent vaccines and given monovalent booster vaccines: an open-label, randomised, controlled, multicentre study. Lancet Infect Dis 2010;10:755-61.

[23] Nasso M, Fedele G, Spensieri F, Palazzo R, Costantino P, Rappuoli R, et al. Genetically detoxified pertussis toxin induces Th1/Th17 immune response through MAPKs and IL-10-dependent mechanisms. J Immunol 2009;183:1892-9.

[24] Hijnen M, van Gageldonk PG, Berbers GA, van Woerkom T, Mooi FR. The Bordetella pertussis virulence factor P.69 pertactin retains its immunological properties after overproduction in Escherichia coli. Protein Expr Purif 2005;41:106-12.

[25] van Gageldonk PG, van Schaijk FG, van der Klis FR, Berbers GA. Development and validation of a multiplex immunoassay for the simultaneous determination of serum antibodies to Bordetella pertussis, diphtheria and tetanus. J Immunol Methods 2008;335:79-89.

[26] Long SS, Welkon CJ, Clark JL. Widespread silent transmission of pertussis in families: antibody correlates of infection and symptomatology. J Infect Dis 1990;161:480-6.

[27] Tomovici A, Barreto L, Zickler P, Meekison W, Noya F, Voloshen T, et al. Humoral immunity 10 years after booster immunization with an adolescent and adult formulation combined tetanus, diphtheria, and 5-component acellular pertussis vaccine. Vaccine 2012;30:2647-53.

[28] Versteegh FG, Mertens PL, deMelker HE, Roord JJ, Schellekens JF, Teunis $\mathrm{PF}$. Age-specific long-term course of IgG antibodies to pertussis toxin after symptomatic infection with Bordetella pertussis. Epidemiol Infect 2005;133: 737-48.

[29] Guiso N, Berbers G, Fry NK, He Q, Riffelmann M, Wirsing von König CH. What to do and what not to do in serological diagnosis of pertussis: recommendations from EU reference laboratories. Eur J Clin Microbiol Infect Dis 2011;30:307-12.

[30] Wiedermann BL. What's wrong with acellular pertussis vaccines. Clin Ther 2013;35:115-8.

[31] Witt MA, Katz PH, Witt DJ. Unexpectedly limited durability of immunity following acellular pertussis vaccination in preadolescents in a North American outbreak. Clin Infect Dis 2012;54:1730-5

[32] Gabutti G, Rota MC. Pertussis: a review of disease epidemiology worldwide and in Italy. Int J Environ Res Public Health 2012;9:4626-38.

[33] Morel S, Denoël P, Godfroid F, Cortvrindt C, Vanderheyde N, Poolman J. Induction of Bordetella pertussis-specific immune memory by DTPa vaccines. Vaccine 2011;29:3449-55.

[34] Denoël P, Poolman J, Carletti G, Veitch K. Effects of adsorption of acellular pertussis antigens onto different aluminium salts on the protective activity in an intranasal murine model of Bordetella pertussis infection. Vaccine 2002;20:2551-5.

[35] Rosado MM, Scarsella M, Pandolfi E, Cascioli S, Giorda E, Chionne P, et al Switched memory B cells maintain specific memory independently of serum antibodies: the hepatitis B example. Eur J Immunol 2011;41:1800-8.

[36] Carollo M, Palazzo R, Bianco M, Pandolfi E, Chionne P, Fedele G, et al. Hepatitis B specific $T$ cell immunity induced by primary vaccination persists independently of the protective serum antibody level. Vaccine 2013;31:506-13.

[37] Libster R, Edwards KM. Re-emergence of pertussis: what are the solutions. Expert Rev Vaccines 2012;11:1331-46. 